\title{
Experimental Teaching Reform of Digital Electronic Technology Based on CDIO
}

\author{
Zhongjian Cai ${ }^{1, *}$, Shibin Tong ${ }^{2}$ \\ ${ }^{1}$ School of Computer Science and Information Engineering, Chongqing Technology and Business University, Chongqing, \\ China \\ ${ }^{2}$ College of Physical Education, Southwest University, Chongqing, China \\ *Corresponding author
}

\begin{abstract}
The CDIO concept was introduced into digital electronic technology experiment teaching, and the reform plan of experiment teaching was discussed from the conceive, design, Implement, operation and examination of experiment. Teaching practice has proved that this experimental teaching mode can not only improve students' practical ability and comprehensive application ability, but also cultivate students' teamwork spirit and communication ability. Students' mastery of the whole knowledge structure, the training of engineering practice ability, and the improvement of team cooperation consciousness have achieved obvious results.

Keywords: CDIO, Experimental teaching, Digital electronic technology, Teaching reform
\end{abstract}

\section{基于CDI0的数字电子技术实验教学改革}

\author{
蔡忠见 ${ }^{1, *}$ ，童世斌
}

${ }^{1}$ 重庆工商大学计算机科学与信息工程学院, 重庆, 中国

${ }^{2}$ 西南大学体育学院, 重庆, 中国

“通讯作者

\section{中文摘要}

将 CDIO 理念引入到数字电子技术实验教学中，从实验的构思、设计、实现、运作、考核几个方面论述 了实验教学的改革方案。教学实践证明, 这种实验教学模式不但能够提高学生的实践能力与综合应用 能力, 同时还能培养学生的团队合作精神与沟通能力。学生对整体知识结构的掌握、工程实践能力的 训练、团队协作意识的提高各个方面, 均取得明显的效果。

关键词: CDIO；实验教学；数字电子技术；教学改革

\section{1. 引言}

CDIO 理念是以麻省理工学院为首的全球几十所著 名大学倡导的现代工程教育框架, 是当前国际工程教育 最新的发展方式, 集构思 (Conceive)、设计 (Design)、 实现 (Implement) 和运作 (Operate) 四个阶段为一体。 让学生进行主动的、实践的、联系的、协作的方式学习 课程。激发学生的学习兴趣, 提高学生解决实际问题的 能力。从而能够在课堂理论学习和实验项目完成的基础 上, 提高工程实践能力。CDIO 理念的核心是 “做中学” 和 “基于项目教育和学习”, 以实际工程项目为载体, 从产品的需求分析、功能设计、生产运行、开发周期等
方面培养学生专业技术知识、个人能力、职业能力和方 法能力, 是一种与产业发展同步的教育模式。作为当前 高等工程教育的一种新型模式, CDIO 以其先进的工程教 育理念、优良的实践可操作性、全面系统的课程体系、 普遍适应的人才培养模式, 赢得了世界上众多高等工程 院校的关注和青睐。

数字电子技术是高校电类专业的基础课程, 是一门 非常重要的专业基础课程, 也是电子类专业的主干课 程, 该课程教学效果的好坏, 直接影响到后续课程的教 学。该课程既有很强的理论性, 也有很强的实践性, 与 工程实际联系紧密。除理论教学外, 必须配以一定的实 践环节, 才能使学生更好地掌握课程内容, 而且实验项 目的内容设计对于整个教学过程中学生能力的培养起 
着至关重要的作用。课程涉及到种类繁多的电路芯片, 这些芯片的内部结构、外部性能和逻辑应用等都随着课 程的推进而越来越复杂。在传统的教学模式中, 教学内 容相对独立, 系统度不够。每学习到一种类型的芯片, 则侧重于该芯片的独立应用, 或者是仅与较少的简单芯 片联合使用完成相关实验内容。由于单个芯片能实现的 功能相对简单而使学生学习起来枯燥乏味, 使学习过程 被动, 自主性不强。传统的以 “验证性” 实验为主的实 验教学模式与实践脱节, 学生无法获得实际的工程经 验, 无法从整体上把握系统开发的思路。可见, 数字电 子技术课程传统的重理论轻实践、强调个人学术能力而 忽视团队协作精神、重视知识学习而轻视开拓创新的实 验教学模式与 CDIO 理念有很大差距。为了提高学生理 论分析与动手实践的能力, 将 CDI0 理念引入到数字电 子技术的实验课程教学中, 对实验教学模式进行改革。

\section{2. 基于CDIO的实验教学内容设计}

根据 CDIO 工程实践理念的要求, 分为四个阶段进 行贯穿式教学模式的教学内容设计。

\section{1. 构思阶段}

教师在授课之初, 首先选择能够贯穿课程内容始终 的综合型实验项目, 布置学习和实验任务。先介绍实验 项目基本功能要求, 并明确需要达到的最终效果, 将实 验内容中所涉及的知识点和理论教学建立起对应关系, 明确各章节知识点在本实验项目中的作用, 将课程全部 知识点进行融会贯通, 从而可以使学生的学习目的更加 明确, 提高了教学效果。要求学生在整个教学周期内要 完成实验的数学建模、电路设计、电路仿真、电路调试、 电路优化、方案交流、结果汇报等全部内容。这一阶段 的主要目标是让学生从整体上了解该课程的主要内容 和基本应用, 以及在先修和后续课中的地位, 激发学生 的学习兴趣。根据实验项目内容, 要 求学生自由组队, 并进行任务分工。在教师引导下, 团队成员集体讨论, 通过深入理解任务内容, 确定所需输入、输出逻辑变量、 系统状态、状态转换关系等, 建立数学模型。可以锻 炼学生的独立思考能力, 查阅资料能力。

\section{2. 设计阶段}

在明确了项目的基本要求后, 教师需要根据教学内 容将项目中需要的基础知识和芯片类型进行分解, 对应 到课程中的相关章节。功能分析环节, 用到课程的数字 逻辑基础知识部分, 包括逻辑变量定义、逻辑函数表述、 真值表; 时序部分主要用到时序逻辑电路状态转移表、 状态转移图等基础知识; 数码显示部分, 主要用到基本 逻辑门电路和组合逻辑电路的译码器芯片。此外的一些 细节和辅助芯片, 在布置实验任务时进行说明, 引导学 生查阅资料，补充完善。

\section{3. 实现阶段}

根据对前两个阶段的了解, 学生在项目实现阶段, 可以有目的地进行各章节的学习。学生进行分组, 组内 成员分任务, 针对不同模块的具体要求, 在课内学习的 基础上，查阅相关资料，提出相应的设计思路，并进行 仿真模拟。将各子任务合并，可以用一些易学易用的仿 真软件如 proteus 等仿真调试, 直到得出正确实验结果, 然后用实际硬件芯片来实现，并用示波器、万用表等相 应工具进行测试, 排查故障, 完成实验项目。在这个过 程中, 学生可以充分理解各芯片在实际应用中的注意事 项等, 锻炼学生工程实践能力。教师主要负责指导学生 进行设计思路的论证, 解答在仿真模拟过程中遇到的问 题并引导学生解决问题。

\section{4. 运作阶段}

团队成员进行项目展示，从设计思路、设计原理、 关键技术、到调试过程遇到的问题及解决方法, 进行全 面的说明。对一个问题从理解到应用, 再到讲解, 进一 步巩固所学知识。这不仅能扩大学生的视野, 而且能培 养团队的协作意识。教师根据实际完成情况和展示效果 对实验项目进行评判。若项目运作后发现仍有不够完善 之处, 学生团队可以在课后进一步设计和实施, 也可以 在此基础上, 通过查阅资料学习更多知识, 设计增加一 些附加功能，使项目功能更完善、更强大。

在数字电子技术实验课程教学中, 结合 protuse 仿 真、硬件实验平台，通过对各章各个知识点的仿真练习 演示, 使学生在巩固学习知识点的同时学会运用各种逻 辑关系、选择不同逻辑功能的芯片实现特定功能的小型 数字系统, 学生的理论知识和实验能力将大大提高。

\section{3. 评价考核}

工程教育模式的实验项目在考核阶段，除了传统的 实验报告外, 更多的比例为采取过程化和多元化的评价 机制。过程化主要体现在对电路设计各个环节均进行评 价, 改变以往仅仅关注任务完成的结果, 而忽视设计过 程的评价模式。从电路设计的基本过程上看，其中的各 个环节是一个递进的过程, 从课题分析、数学建模、电 路设计、电路仿真、电路连接、电路测试到电路完善。 每个环节不仅对于最后成果的呈现非常重要, 而且对下 一个环节如何进行起到至关重要的作用。因此, 根据各 个阶段性任务是否符合要求, 或者在符合要求的前提下 完成的质量情况, 给予一定比例的分数。多元化包括 评价主体的多元化和评价对象的多元化，打破传统单一 的由老师评价学生完成的状况和只评价个人任务完成 的状况, 加入组内评价和不同组之间的评价。既关注学 生的基本技能及基础知识掌握情况, 也关注学生的创新 能力; 既关注项目最终的完成情况, 也关注项目实施中 的团队协作能力及个人的职业素养; 既有教师的评价, 
也有学生间的互评等。将任务完成情况和团队合作及个 人在团队中贡献度结合起来, 从而避免了仅评价实验报 告的片面性, 使最终评价更加合理、完善, 极大地提高 了学生在学习和实验过程中的主观能动性。

通过对 “数字电子技术” 实验教学模式的改革, 增 加创新型工程实践项目, 使学生在完成项目的过程中了 解知识点如何应用, 学生思考问题和解决问题的能力得 到逐步提高。学生从信息收集、制定计划、选择方案到 实施方案的过程中全程参与, 使学生从深度和广度上扩 充知识, 激发了自身的学习积极性和创造性, 有助于创 新能力的培养。

\section{4. 结束语}

对数字电子技术课程实验教学模式进行改革, 引入 CDI0 理念的教学模式, 以学生为教育主体, 以实际工程 项目为载体, 培养学生构思、设计、实现、运作的能力, 对激发学生学习兴趣、明确学习方向、转变学习态度、 提高专业基础水平和团队合作意识以及提高教学效果 等方面发挥出了明显的积极作用。引入 CDIO 理念的教 学模式不仅能提升实验教学效果, 对理论教学也有积极 作用, 使学生对基本理论知识有更完整和更深层次的理 解, 通过依托实验项目对教学内容的分解, 激发了学生 的学习兴趣, 有利于教学过程的开展, 并在此基础上培 养学生的创新精神、系统性思维能力、工程实践能力和 团队协作意识, 很好地保证了教学质量和教学效果。

\section{REFERENCES}

[1] JI Xiu-xia, Research on "Digital Electronic Technology" Experimental Teaching Model Based on CDIO, Education Teaching Forum, No.27, Jul. 2019.

[2] Ding Shu-yan, Li Shi-bao, Cai Li-ping, Zhang Xi-ling,Exploration and practice of digital logic circuit teaching reform based on CDIO, Education Mordernization, No.48,Nov.2018.

[3] Wu xiao-die, Teaching reform of power electronic technology based on CDIO mode and Internet, Think tank Era, Nov.2019.

[4] DING Shu-yan, LI Shi-bao, GU Li-hong, Exploration on the Reform of Digital Logic Experiment Teaching Based on Fusion of CDIO, Education Teaching Forum, No.25, Jun. 2019.

[5] Zhong-jian CAI, Shi-bin TONG, Experimental teaching reform of digital electronic technology based on Proteus simulation software, 2019 2nd International Conference on Education Reform, Management and Applied Social Science(ERMASS 2019). 